\title{
Turbulent latent and sensible heat flux in the presence of evaporative droplets
}

\author{
Brian Helgans ${ }^{\mathrm{a}}$, David H. Richter ${ }^{\mathrm{a}, *}$ \\ ${ }^{a}$ University of Notre Dame, Department of Civil and Environmental Engineering and Earth \\ Sciences, Notre Dame, IN 46556
}

\begin{abstract}
Direct numerical simulations of an Eulerian-based carrier phase are performed which are two-way coupled in momentum and energy to Lagrangian droplets within a Boussinesq-type incompressible formulation, where the droplets are allowed to evaporate and condense and are thus coupled to the vapor field of the carrier phase. Turbulent planar Couette flow is simulated under varying boundary forcings to understand the degree to which evaporating droplets modify vertical fluxes of energy in horizontally homogeneous systems. In particular, the separate influences on both sensible and latent heat are substantial but opposite in sign, and the local relative humidity can result in droplet-induced heat or moisture fluxes which counteract the prescribed background gradients. The influence of droplet Stokes number is also considered, where it is shown that both clustering and turbophoresis play important roles in determining the magnitude of the droplet-induced sensible and latent heat fluxes.

Keywords: evaporating droplets, two-way coupling, heat fluxes, direct numerical simulation
\end{abstract}

\section{Introduction}

In many industrial and natural environments, the addition of suspended solid or liquid particles within a turbulent carrier gas phase can result in sig-

*Corresponding author: 156 Fitzpatrick Hall, Notre Dame, IN, 46556; 1-574-631-4839; David.Richter.26@nd.edu

Preprint submitted to Elsevier

September 22, 2015

(C) 2015. This manuscript version is made available under the Elsevier user license http://www.elsevier.com/open-access/userlicense/1.0/ 
nificant modifications to the bulk statistics of velocity, temperature, and other scalar quantities throughout the system. For instance the phenomenon of turbulence modulation (Balachandar and Eaton, 2010), where solid particles modify carrier phase motions through momentum coupling, can for example result in altered velocity fluctuation profiles and Reynolds stresses in turbulent flows near walls depending on the size and concentration of the particulate phase (Kaftori et al., 1998; Righetti and Romano, 2004; Kulick et al., 1994). In applications ranging from industrial powder transport to the formation of riverbed ripples, this dynamic coupling mechanism between phases requires careful attention if bulk-scale models are to be faithfully developed (Crowe, 2000).

For numerically modeling particulate-laden flows where elements of the dispersed phase remain small compared to the smallest turbulent length scales of the flow, the Lagrangian point-particle approach has been used extensively in combination with direct numerical simulation (DNS) of the carrier phase to study particle dispersion and momentum coupling in a wide variety of isotropic (Elghobashi and Truesdell, 1993; Boivin et al., 1998) and wall-bounded (Zhao et al., 2013; Soldati and Marchioli, 2009; Dritselis and Vlachos, 2011) flows. Details regarding processes such as preferential concentration or clustering (Richter and Sullivan, 2014a; Bernardini et al., 2013; Sardina et al., 2012; Rouson and Eaton, 2001) and the effects of particle Stokes number (Lee and Lee, 2015; Richter and Sullivan, 2013; Ferrante and Elghobashi, 2003) have been continually uncovered, which in turn have provided insight into physical mechanisms which are often difficult to observe or measure experimentally.

Beyond momentum coupling, however, a dispersed phase, depending on its material properties, also possesses the ability to modify the thermodynamic characteristics of the carrier phase flow, which has important implications for instance in systems where droplet size evolution is critical, such as within combustion chambers (Miller and Bellan, 1999) or during cloud formation (Vaillancourt and Yau, 2000). Owing to the larger degree of complexity in considering both two-way dynamic and thermodynamic coupling, less attention has been focused on understanding the feedbacks and carrier phase modifications in the 
fully coupled system, particularly from an experimental perspective. Studies are beginning to emerge, however, which, as in the case of two-way momentum coupling, offer progress in understanding energy and mass coupling mechanisms in particulate systems.

Considering only heat coupling - i.e., non-evaporating particles whose temperature differences with the ambient drive sensible heat transfer between phases - Shotorban et al. (2003) performed DNS of transient homogeneous turbulent shear flow laden with small, two-way coupled particles. They monitored the temperature variance budget and turbulent heat fluxes under varying particle size, concentration, and specific heat ratio, and found a general decrease of carrier phase temperature variance and turbulent heat flux with the addition of particles. Similarly, Zonta et al. (2008) and Zonta et al. (2011) studied turbulent channel flow dispersed with low concentrations of small, thermally coupled inertial particles, and illustrated the dual function of particles: altering cross-channel heat fluxes through both a modification to the mean temperature gradient as well as the Reynolds stress ejection events near the wall.

Kuerten et al. (2011) find similar results for thermally coupled particles in a turbulent channel flow, generally finding an enhancement of nondimensional cross-channel heat transfer depending on the dispersed phase characteristics. They again highlight the dual influence of particles through momentum and thermal coupling by breaking the total heat flux into the laminar, turbulent, and particle-induced components - a process that was also used by Richter and Sullivan (2014b), who analyzed the heat flux budget for thermally coupled particles in turbulent planar Couette flow, and who demonstrated an efficient heat transfer mechanism where particles of Stokes number near unity collect in the ejection regions typically responsible for turbulent fluxes in unladen conditions.

Studies that consider full thermodynamic coupling, where droplet evaporation is included in addition to momentum and energy coupling, are fewer in number. Mashayek (1998) performed an extensive analysis of the effects of evaporating droplets in weakly compressible homogeneous shear flows, and illustrates the significant modifications to both kinetic and internal energy evolution in the 
system, which is different when considering evaporating versus non-evaporating droplets. Miller and Bellan (1999) similarly consider evaporating droplets in weakly compressible environments, focusing on the mixing between a laden and unladen stream of gas. Particle clustering and entrainment of relatively unsaturated gas results in a broadening of the droplet size distribution, while at the same time lowering the kinetic and thermal energy of the downstream gas phase.

Recently, Russo et al. (2014) outlined an incompressible formulation of thermodynamically coupled droplets in turbulent channel flow. They found significant modifications to the mean temperature and moisture profiles, and, using the same breakdown of heat transport as used by Kuerten et al. (2011), demonstrated a substantial increase in the bulk nondimensional cross-channel Nusselt number after adding thermodynamically coupled droplets - an effect driven by particle/ambient temperature differences, which is enhanced when the droplets are allowed to evaporate. Subsequent studies by Bukhvostova et al. (2014b) and Kuerten and Vreman (2015) illustrate the influence of relative humidity and particle collisions, respectively.

The current study aims to extend this overall understanding of energy and flux modification by simulating droplet-laden turbulent planar Couette flow and evaluating the components of the wall-normal fluxes of sensible and latent heat. Our focus is broadly motivated by droplets suspended over marine surfaces, and specifically whether or not their presence can enhance or attenuate upward energy fluxes. Since evaporating droplets ultimately provide the link between sensible and latent heat transfer, understanding the basic yet highly coupled effects of evaporative cooling and enhanced moisture transport on total energy transfer are necessary for fully assessing their ability to modify energy transport.

As will be noted throughout, the simulations presented herein are generally similar to those of Russo et al. (2014) and Bukhvostova et al. (2014b), but extends the analysis to probe specific thermodynamic feedback mechanisms between the droplets and the surrounding flow. We focus particularly on the breakdown of total enthalpy flux into the sensible and latent components, with 
emphasis on the difference between droplet effects on each component independently versus the overall droplet effect on the sum - a breakdown with implications on accurate model development. We first present a numerical model in section 2 which solves a two-way coupled set of equations where droplets and the surrounding turbulent flow are coupled in momentum, energy, and water vapor mass. We then present the results of a series of direct numerical simulations, where boundary forcings are varied in a systematic way that highlight unique cross-phase thermodynamic couplings present in the system. It is this regard that the current study differs significantly from that of Russo et al. (2014), and a breakdown of both sensible and latent energy flux is provided in section 3.1. Section 3.2 shows the variation of cross-channel enthalpy fluxes as a result of boundary forcing and section 3.3 illustrates the influence of droplet size on these findings. Finally, section 4 summarizes our conclusions.

\section{Numerical model}

The current study aims to better understand thermodynamic coupling in turbulent flows under the influence of small (smaller than the Kolmogorov length scale of the flow), relatively high-density saline water droplets. Thus, this work employs the combined Eulerian-Lagrangian strategy of performing DNS of the carrier phase (air) while representing spherical saline water droplets as point particles, each dynamically and thermodynamically coupled to the surrounding flow. The numerical model is therefore similar to those of Mashayek (1998), Miller and Bellan (1999), and Russo et al. (2014).

\subsection{Carrier phase}

The equations governing conservation of energy, moisture, momentum, and mass of the ambient (carrier phase) fluid are solved via DNS. In the context of moist air, we invoke a Boussinesq-type approximation by assuming that the addition of water vapor does not appreciably modify the carrier phase density. Thus, $\rho_{\text {gas }} \approx \rho_{a}$, where $\rho_{\text {gas }}$ is the total gas mixture density (air plus water 
vapor) and $\rho_{a}$ is the dry air density. This approximation also assumes that temperature variations in the system are not large enough to produce appreciable density variations. For the conditions specified in the following simulations, these approximations hold true within $1 \%$. Note that, since their primary motivation includes combustion processes (where these approximations do not necessarily hold), the Eulerian-Lagrangian simulations of both Mashayek (1998) and Miller and Bellan (1999) utilize a fully compressible formulation and do not make this constant density assumption. Russo et al. (2014) meanwhile, uses an incompressible formulation similar to that used in the current study. The similarities and differences will be discussed in more detail below. We also note that given the small temperature and humidity ranges specified in the present simulations (and in accordance with the standard Boussinesq assumption), we assume that temperature and/or humidity-dependent thermodynamic properties such as viscosity or thermal diffusivity are unnecessary. In certain conditions these effects can be significant (Zonta et al., 2012; Zonta and Soldati, 2014), but Russo et al. (2014) for example show that the influence of a humidity-dependent thermal conductivity is small in similar setups.

Mass conservation under these assumptions therefore yields a divergence-free condition for the carrier phase velocity field:

$$
\frac{\partial u_{i}}{\partial x_{i}}=0
$$

which is enforced by solving a pressure Poisson equation at each Runge-Kutta stage.

Again under the incompressible approximation and neglecting buoyancy forces, momentum conservation of the carrier phase yields:

$$
\frac{\partial u_{i}}{\partial t}+u_{j} \frac{\partial u_{i}}{\partial x_{j}}=-\frac{1}{\rho_{a}} \frac{\partial p}{\partial x_{i}}+\nu_{a} \frac{\partial^{2} u_{i}}{\partial x_{j} \partial x_{j}}+\frac{1}{\rho_{a}} S_{i}^{m}
$$

where $\nu_{a}=\mu_{a} / \rho_{a}$ is the carrier phase kinematic viscosity and $S_{i}^{m}$ is the momentum coupling source due to the presence of the particles, to be defined in section 2.3 . 
By conservation of vapor mass, the specific humidity field $q$ is governed by:

$$
\frac{\partial q}{\partial t}+u_{j} \frac{\partial q}{\partial x_{j}}=D_{v} \frac{\partial^{2} q}{\partial x_{j} \partial x_{j}}+S^{q}
$$

The vapor diffuses into dry air with an assumed constant diffusivity $D_{v}$, and is coupled to the droplets by the source/sink term $S^{q}$. Note that we use the term "specific humidity" interchangeably with "mixing ratio" since we have assumed that $\rho_{g a s} \approx \rho_{a}$, and thus $q\left(x_{i}, t\right)$ is defined herein as $q=\rho_{v} / \rho_{a}$, where $\rho_{v}\left(x_{i}, t\right)$ is the local vapor density (or absolute humidity) which can vary in space and time, while $\rho_{a}$ is constant.

Under the assumption of constant mixture density and with the presence of thermodynamically coupled particles, energy conservation must be handled carefully, as in for example Russo et al. (2014). Ultimately, the equation governing the air temperature is given by:

$$
\frac{\partial T}{\partial t}+u_{j} \frac{\partial T}{\partial x_{j}}=\alpha \frac{\partial^{2} T}{\partial x_{j}^{2}}+\frac{1}{\rho_{a} c_{p, a}}\left(S^{h, c o n v}+S^{h, e v a p}\right),
$$

where $T$ is the air temperature, $c_{p, a}$ is the (assumed constant) specific heat of dry air at constant pressure, and $\alpha=k_{T} / \rho_{a} c_{p, a}$ is the (assumed constant) thermal diffusivity of the carrier phase, where $k_{T}$ is the thermal conductivity of air. As is commonly done, this expression neglects internal energy generation from dissipation of kinetic energy. The influence of the droplets is split into two terms: $S^{h, c o n v}$ represents heat exchange between the dispersed and carrier phases due to

convective heat transfer, while $S^{h, e v a p}$ accounts for the sensible heat exchange occurring between phases due to evaporation (i.e., mixing of vapor and air at different temperatures). Details regarding this equation's development are provided in the appendix.

\subsection{Dispersed phase}

Conservation of mass, momentum, and energy of individual water droplets can be enforced to yield equations governing the particle location, velocity, temperature, and radius (or mass). The particle position is dictated purely by advection:

$$
\frac{d x_{p, i}}{d t}=v_{p, i}
$$


where $x_{p, i}$ is an individual particle location and $v_{p, i}$ is its velocity. Assuming the particle to fluid density ratio $\rho_{p} / \rho_{a}$ is large and neglecting gravity, the acceleration of the droplet is governed solely by an empirically modified Stokes drag force Maxey and Riley (1983); Clift et al. (1978):

$$
\frac{d v_{p, i}}{d t}=\left(1+0.15 \operatorname{Re}_{p}^{0.687}\right) \frac{1}{\tau_{p}}\left(u_{a, i}-v_{p, i}\right),
$$

where $R e_{p}$ is the droplet Reynolds number $R e_{p}=d_{p}\left|u_{a, i}-v_{p, i}\right| / \nu_{a}\left(d_{p}\right.$ is the droplet diameter), the surrounding air velocity at the particle location is given by $u_{a, i}$, and $\tau_{p}=\rho_{p} d_{p}^{2} / 18 \rho_{a} \nu_{a}$ is the standard Stokes timescale of the particle, updated at each time step based on the current $d_{p}$ and $\rho_{p}$. Note that the momentum rate-of-change term associated with the rate of change of particle mass $\left(v_{p, i} \dot{m}\right.$ where $\dot{m}=d m_{p} / d t$ is the condensation rate) cancels with the outward momentum flux contained by the evaporated water vapor. Note also that we consider saline water droplets, so that the variable droplet density $\rho_{p}$ is not equal to the constant density of pure water $\rho_{w}$ unless the salinity is zero.

For this study, we neglect gravitational forces on droplet trajectories in order to analyze energy transfer in the simplest case possible, and not necessarily because we are interested specifically in regimes where gravitational forces are negligible. Likewise, we neglect particle-particle collisions for simplicity, and justify this by maintaining a bulk volume fraction of $\phi_{V}=1.1 \times 10^{-4}$ throughout all simulations in order to restrict the analysis to regimes where two-way coupling is likely sufficient (Balachandar and Eaton, 2010; Elghobashi, 1994). We thus assume that enhanced local droplet concentrations for example due to turbophoresis or local clustering occur infrequently enough such that they do not substantially modify the results presented here. Based on our own preliminary experiments as well as previous studies (e.g., Yamamoto et al. (2001) and Kuerten and Vreman (2015)), we can speculate that one of the primary effects of including particle-particle collisions would be to reduce local concentrations, particularly near the walls as resulting from turbophoresis, which can actually enhance many of the mechanisms identified in this work due to a consequent rise in particle concentration in the domain interior. The effects of gravity and 
consequent droplet resuspension as well as particle-particle collision/coalescence are topics of ongoing work.

To model droplet evaporation, we use a microphysical model outlined in Pruppacher and Klett (1997), which accounts for both the effects of curvature and salinity on water evaporation from the droplet surface. In this way the present model deviates from that of Russo et al. (2014), Miller and Bellan (1999), and Mashayek (1998). In this model, mass transfer is driven by the difference between the ambient specific humidity at the droplet location $\left(q_{a}\right)$ and the specific humidity immediately adjacent to the droplet surface $\left(q_{*}\right)$ using a convective mass transfer coefficient $\bar{h}_{m}$ which can be expressed through a dimensionless droplet Sherwood number $S h_{p}=d_{p} \bar{h}_{m} / D_{v}$. Assuming a spherical droplet, the rate of change of mass $\dot{m}$ is given by:

$$
\dot{m}=\frac{d m_{p}}{d t}=4 \pi r_{p}^{2} \rho_{w} \frac{d r_{p}}{d t},
$$

and can thus be related to its change in radius. Therefore an equation governing the particle radius $r_{p}$ is given by:

$$
\frac{d r_{p}}{d t}=\bar{h}_{m} \frac{\rho_{a}}{\rho_{w}}\left(q_{a}-q_{*}\right)=\frac{1}{9} \frac{S h_{p}}{S c} \frac{\rho_{p}}{\rho_{w}} \frac{r_{p}}{\tau_{p}}\left(q_{a}-q_{*}\right) .
$$

As noted above, $\rho_{w}$ is the density of pure water, while $\rho_{p}$ is the density of the saline droplet. $S c$ refers to the vapor Schmidt number, which relates the vapor diffusivity to the kinematic viscosity of the air: $S c=\nu_{a} / D_{v}$. The relationship for the Sherwood number is given by the empirical expression of Ranz and Marshall (1952):

$$
S h_{p}=2+0.6 \operatorname{Re}_{p}^{1 / 2} S c^{1 / 3} .
$$

The specific humidity adjacent to the droplet surface, $q_{*}$, dictates whether or not the droplet will evaporate, and is a function of the droplet temperature, salinity, and radius. An extensive derivation in Pruppacher and Klett (1997) (p. 509) yields an expression which does not merely assume that $q_{*}$ is the saturation 
humidity at the droplet temperature:

$$
\begin{aligned}
q_{*} & =\frac{M_{w} e_{*}}{R_{u} T_{p} \rho_{a}} \\
& =\frac{M_{w}}{R_{u} T_{p} \rho_{a}} e_{s, a} \exp \left[\frac{L_{v} M_{w}}{R_{u}}\left(\frac{1}{T_{a}}-\frac{1}{T_{p}}\right)+\frac{2 M_{w} \sigma}{R_{u} \rho_{w} r_{p} T_{p}}-\frac{I \Phi_{s} m_{s}\left(M_{w} / M_{s}\right)}{m_{w}}\right] .
\end{aligned}
$$

The first equality in equation 10 relates the surface specific humidity $q_{*}$ to the surface vapor pressure $e_{*}$ via the ideal gas law, and therefore $M_{w}$ represents the molecular weight of water and $R_{u}$ is the universal gas constant. The second equality relates the surface vapor pressure $e_{*}$ to the ambient saturated vapor pressure at the droplet location, $e_{s, a}$, and other droplet characteristics. To approximate the Clausius-Clapeyron equation and retrieve $e_{s, a}$, a modified version of the Magnus relation (Alduchov and Eskridge, 1996) is used to determine the saturation vapor pressure $e_{s, a}$ evaluated at $T_{a}$ :

$$
e_{s, a}\left(T_{a}\right)=610.94 \exp \left(\frac{17.625 T_{a}}{243.04+T_{a}}\right),
$$

where $T_{a}$ is provided in Celsius and $e_{s, a}$ is provided in Pa.

The latent heat of vaporization of water in air, $L_{v}$, the surface tension of a flat air-water interface $\sigma$, and the osmotic coefficient $\Phi_{s}$ are all assumed to be constant. In practical terms, the effect of droplet curvature (second term within the exponential in equation 10) is to enhance evaporation at small $r_{p}$ while salinity (third term within the exponential in equation 10) acts to suppress evaporation when the droplet becomes too saline. The salinity effect offers a cut-off mechanism for the evaporation process which would not exist otherwise, thus removing the possibility of droplets reaching a diameter of 0 .

The total mass of salt in the droplet is given by $m_{s}$, the mass of pure water is $m_{w}$, and the molecular weight of salt is given by $M_{s}$. Salt dissociates into two ions, making the coefficient $I=2$. With the known gain/loss of pure water determined by equation 8 , the droplet salinity is updated algebraically based on the prescribed initial salinity and corresponding particle volume assuming no salt mass is lost during the evaporation process. Note that in the current simulations, the effects of both curvature and salinity are very small. 
Finally, conservation of energy applied to a droplet yields an equation governing its temperature $T_{p}$. As in Russo et al. (2014), we have neglected the kinetic energy of the droplet phase in comparison to its internal energy. We also assume that heat conduction within the droplet is instantaneous compared to the convection at the surface, and thus the droplet has a uniform temperature throughout. As a result, the internal energy of a droplet will change based on two processes: convective sensible heat transfer driven by temperature differences between $T_{p}$ and the ambient air temperature at the droplet location $T_{a}$, and latent heat transfer due to the droplet evaporation:

$$
\begin{aligned}
\frac{d T_{p}}{d t} & =\frac{\dot{Q}_{c o n v}}{\rho_{w} V_{d} c_{L}}+\frac{\dot{Q}_{l a t}}{\rho_{w} V_{d} c_{L}} \\
& =\left[-\frac{1}{3} \frac{N u_{p}}{\operatorname{Pr}} \frac{c_{p, a}}{c_{L}} \frac{\rho_{p}}{\rho_{w}} \frac{1}{\tau_{p}}\left(T_{p}-T_{\infty}\right)\right]+\left[3 L_{v} \frac{1}{r_{p} c_{L}} \frac{d r_{p}}{d t}\right] .
\end{aligned}
$$

where in the first equality $V_{d}$ is the droplet volume, $c_{L}$ is the (constant) liquid water specific heat, $\dot{Q}_{c o n v}$ is the convective heat transfer rate into the droplet, and $\dot{Q}_{l a t}$ is the latent heat transfer rate. The use of the pure water density $\rho_{w}$ instead of $\rho_{p}$ reflects the fact that we assume that the water holds all of the droplet internal energy (as opposed to the salt).

In the second equality, the first term in brackets corresponds to $\dot{Q}_{c o n v}$ while the second bracketed term corresponds to $\dot{Q}_{l a t}$. Convective heat transfer is parameterized through a bulk heat transfer coefficient $\bar{h}_{e}$ which is determined through an empirical expression for the droplet Nusselt number (Ranz and Marshall, 1952):

$$
N u_{p}=\frac{\bar{h}_{e} d_{p}}{k_{T}}=2+0.6 \operatorname{Re}_{p}^{1 / 2} \operatorname{Pr}^{1 / 3} .
$$

Here, the Prandtl number $\operatorname{Pr}=\nu_{a} / \alpha$ is defined as the ratio of kinematic viscosity of the air to the constant thermal diffusivity $\alpha$.

Note that the latent heat transfer term is merely the rearranged version of the original expression $\dot{Q}_{l a t}=L_{v} \dot{m}$ after assuming sphericity, where $\dot{m}$ is the rate of vapor mass entering a droplet.

Thus, given the ambient air velocity $u_{a, i}$, temperature $T_{a}$, and specific humidity $q_{a}$, all of which are interpolated to the droplet location using sixth order 
Lagrange polynomials, equations $5,6,8$, and 12 are solved for the particle position, velocity, radius, and temperature, respectively. These equations are integrated in time using the same RK3 scheme as used for the carrier phase.

\subsection{Two-way coupling}

The droplets are two-way coupled to the carrier phase through the source terms $S_{i}^{m}, S^{q}, S^{h, c o n v}$, and $S^{h, \text { evap }}$ which appear in equations 2,3 , and 4 . In general, these coupling terms are computed by linearly projecting the individual particle sources onto the eight Eulerian computational nodes which make up the grid cell surrounding a given droplet.

For momentum, the rate of change of momentum of the particle $d\left(m_{p} v_{p, i}\right) / d t$ results from a drag component (the right hand side of equation 6) and the change in momentum due to gain/loss of water mass, which must appear in the coupling term because the vapor contains momentum, albeit a very small amount. Thus the source term $S_{i}^{m}$ is computed as:

$$
S_{i}^{m}=-\sum_{\beta=1}^{\mathcal{N}_{p}} \frac{w_{\beta}}{\Delta V}\left(m_{p} \frac{d v_{p, i}}{d t}+\dot{m} v_{p, i}\right)_{\beta},
$$

where the contribution from all $\mathcal{N}_{p}$ particles residing across all eight grid cells which share a carrier phase node are summed. The contribution of each particle $\beta$ is weighted linearly to the node via $w_{\beta}$, and $\Delta V$ is the volume of the grid cell where the particle resides. The particle acceleration $d v_{p, i} / d t$ is given by equation 6 , and the mass condensation rate $\dot{m}$ is related to the rate of change of radius as shown in equation 7 .

In a similar way, vapor coupling is enforced by summing all evaporated water vapor from droplets in the vicinity of each node into the humidity field:

$$
S^{q}=-\sum_{\beta=1}^{\mathcal{N}_{p}} \frac{w_{\beta}}{\Delta V}\left(\frac{\dot{m}}{\rho_{a}}\right)_{\beta},
$$

where again the condensation rate $\dot{m}$ is computed using equation 7 and the right hand side of equation 8 . 
As noted above, several subtleties exist which are associated with casting the thermodynamically coupled system in an incompressible formulation, and many of these points are addressed in the appendix. Russo et al. (2014) carefully derive an incompressible equation set, and a short time later Bukhvostova et al. (2014a) show that the incompressible formulation very closely matches the fully compressible formulation for low-Mach-number flows. In the present setup, we make a Boussinesq-type approximation which assumes that $\rho_{\text {gas }}=\rho_{a}$, which is slightly different than the approximation of Russo et al. (2014), who assume that both $\rho_{a}$ and $\rho_{v}$ can vary in space and time, while their sum $\rho_{\text {gas }}$ remains constant (see their equation 2.1).

Under our approximation, the coupling terms $S^{h, c o n v}$ and $S^{h, e v a p}$ are computed by:

$$
\begin{gathered}
S^{h, \text { conv }}=-\sum_{\beta=1}^{\mathcal{N}_{p}} \frac{w_{\beta}}{\Delta V}\left(\dot{Q}_{\text {conv }}\right)_{\beta}, \\
S^{h, \text { evap }}=-\sum_{\beta=1}^{\mathcal{N}_{p}} \frac{w_{\beta}}{\Delta V}\left(\dot{m}\left(c_{p, v} T_{a}-c_{p, v} T_{p}\right)\right)_{\beta},
\end{gathered}
$$

where $\dot{Q}_{c o n v}$ is given in the second equality of equation 12 and $c_{p, v}$ is the specific heat of vapor at constant pressure. The physical meaning of $S^{h, c o n v}$ is the sensible heat exchange driven by temperature differences between the droplet and ambient, while $S^{\text {hevap }}$ accounts for the sudden mixing of vapor with temperature $T_{p}$ with the surrounding air at $T_{a}$, potentially changing its temperature. As will be shown later (and is demonstrated in Russo et al. (2014)), this term is very small.

\subsection{Numerical setup}

The simulation setup is nearly identical to that used by our group in previous investigations of particle-turbulence momentum and heat coupling which did not consider the full thermodynamic coupling (Richter and Sullivan, 2013, 2014b; Richter, 2015). The simulated flow is turbulent planar Couette flow, which develops between two infinite planes moving with equal and opposite velocity $U_{0} / 2$ separated by a distance $H$. This flow provides a convenient framework 
for evaluating wall-normal fluxes, and offers large-scale structures which are not present in the more common turbulent channel flow (Komminaho et al., 1996), which can be very useful for interpreting dispersed phase dynamics (Bernardini et al., 2013). The bulk Reynolds number is $R e_{b}=U_{0} H / \nu_{a}=8085$ which corresponds to a friction Reynolds number of $\operatorname{Re}_{\tau}=u_{\tau}(H / 2) / \nu_{a} \approx 120$. The friction Reynolds number varies between cases due to the momentum coupling enforced between phases.

The code is pseudospectral (with antialiasing) in the periodic $x$ and $y$ directions and uses second order finite differencing in the inhomogeneous, wall-normal $z$ direction. Grid-converged simulations are performed on a domain spanning $[2 \pi H, 2 \pi H, H]$ in the $[x, y, z]$ directions with a grid resolution of $\left[N_{x}, N_{y}, N_{z}\right]=$ $[128,256,128]$. With our code, this particular grid resolution has been verified to provide carrier phase turbulence statistics in good agreement with past direct numerical simulations of Couette flow (Komminaho et al., 1996), and tests compared to the particle-laden channel flow benchmark case of Marchioli et al. (2008) compare favorably as well. Algebraic grid stretching is used in the $z$ direction, where $\Delta z_{\text {min }}^{+}$(where the superscript [ ] ${ }^{+}$refers to viscous scaling), the minimum grid spacing in the wall-normal direction is approximately equal to 0.8 . The bulk of the simulations are done with particles of $d_{p}^{+}=0.6$, while section 3.3 considers droplets with diameters corresponding to $d_{p}^{+}=0.2$ and $d_{p}^{+}=1.2$ (the latter case thus considers particles whose diameter slightly exceeds the grid spacing at the wall, but this is only true in the lowest 14 grid cells). Time is advanced with a low-storage, third-order Runge-Kutta (RK3) scheme (Spalart et al., 1991).

The boundary conditions are specified as follows. The carrier phase velocity is no-slip at the upper and lower walls, with periodicity enforced in the homogeneous $x$ and $y$ directions. Dirichlet conditions for temperature and moisture are prescribed at the upper and lower walls, in various combinations to be presented shortly. The combination of temperature and humidity specified at each wall corresponds to a total enthalpy (computed via $\left.h_{\text {gas }}=\rho_{a} c_{p, a} T+\rho_{a} q\left(c_{p, v} T+h_{v}^{0}\right)\right)$, whose difference $\Delta h$ between the bottom 
and top walls drives the total energy transfer.

For the dispersed phase, particle collisions with the wall are perfectly elastic, and the collision location is enforced to occur at a distance $h_{\text {collision }}^{+}=1$ from either wall, regardless of the droplet diameter. The reasoning behind this collision scheme is to homogenize the impact of the particle boundary conditions, such that no particle size has a stronger or weaker tendency to become "stuck" in the region immediately adjacent to the wall, consequently influencing the results merely through changing near-wall concentrations. Furthermore, in the context of particles which are meant to represent liquid droplets, elastic collisions at a wall are an unphysical approximation to begin with, and the dynamics of the point particle model at a wall are inherently flawed since, among other things, forces such as lift are neglected. In this sense, we treat elastic boundary conditions for the dispersed phase as merely a means to keep the droplets suspended in the interior of the flow. Other particle boundary conditions, such as allowing the droplets to absorb and/or re-deposit from the surface (Zonta et al., 2013; Mito and Hanratty, 2006), could potentially add more realistic conditions for water droplets transported in wall-bounded turbulence, but again in this study we wish to minimize the impact of droplet boundary parameterizations and instead focus on thermodynamic influences given a suspended concentration within the channel interior.

Finally, the constant parameters used throughout this study and introduced throughout the previous two sections are provided in table 1 . Nearly all values are chosen to represent saline water droplets in air, thus specifying all specific heats, densities, salinity parameters, and the dimensionless Schmidt and Prandtl numbers - these are all presented in table 1. Furthermore a reference temperature of $T_{r e f}=26^{\circ} \mathrm{C}$ is used to compute the constant latent heat of vaporization $L_{v}$ using the relationship of Andreas (1995) evaluated at $T_{r e f}$ :

$$
L_{v}=L_{v}\left(T_{r e f}\right)=\left(25.00-0.02274 T_{r e f}\right) \times 10^{5},
$$

where $T_{r e f}$ is provided in degrees Celsius and $L_{v}$ has units of $\mathrm{J} \mathrm{kg}^{-1}$. With this 


\begin{tabular}{c|c} 
Parameter & Value \\
\hline \hline$R e_{b}$ & 8085 \\
$S c=\nu_{a} / D_{v}$ & 0.615 \\
$P r=\nu_{a} / \alpha$ & 0.715 \\
$\rho_{a}$ & $1.1 \mathrm{~kg} \mathrm{~m}^{-3}$ \\
$\rho_{w}$ & $1000 \mathrm{~kg} \mathrm{~m}^{-3}$ \\
$\nu_{a}$ & $1.537 \times 10^{-5} \mathrm{~m}^{2} \mathrm{~s}^{-1}$ \\
$c_{p, a}$ & $1006 \mathrm{~J} \mathrm{~K}^{-1} \mathrm{~kg}^{-1}$ \\
$c_{p, v}$ & $1952 \mathrm{~J} \mathrm{~K}^{-1} \mathrm{~kg}^{-1}$ \\
$c_{L}$ & $4179 \mathrm{~J} \mathrm{~K}^{-1} \mathrm{~kg}^{-1}$ \\
$L_{v}$ & $2441 \times 10^{3} \mathrm{~J} \mathrm{~kg}^{-1}$ \\
$h_{v}^{0}$ & $2383 \times 10^{3} \mathrm{~J} \mathrm{~kg}^{-1}$ \\
$\sigma$ & $7.28 \times 10^{-2} \mathrm{~N} \mathrm{~m}^{-1}$ \\
$\Phi_{s}$ & 1.093 \\
$I$ & 2 \\
$R_{u}$ & $8.314 \mathrm{~J} \mathrm{~mol}{ }^{-1} \mathrm{~K}^{-1}$ \\
$M_{w}$ & $0.018 \mathrm{~kg} \mathrm{~mol}^{-1}$ \\
$M_{s}$ & $0.0584 \mathrm{~kg} \mathrm{~mol}^{-1}$ \\
$U_{0}$ & $1.59 \mathrm{~ms}^{-1}$ \\
$H$ & $0.04 \mathrm{~m}$
\end{tabular}

Table 1: Constants specified in the numerical model 
value of $L_{v}$, the reference enthalpy is computed via:

$$
h_{v}^{0}=L_{v}-\left(c_{L}-c_{p, v}\right)\left(T_{r e f}+273.15\right),
$$

where $c_{L}$ is the specific heat of liquid water. Equation 19 merely expresses that the reference enthalpy is the latent heat evaluated at $T=0 \mathrm{~K}$. Aside from material properties and reference states, the primary quantities with significant influence on the physical processes targeted by this study are the flow Reynolds number, which is limited by computational requirements in the DNS framework; the particle initial Stokes number, which is determined by their initial size and density and provides an indication of the relative inertia of each droplet; and the boundary conditions of the flow, which force heat and moisture transfer across the domain. The details of how these parameters are chosen will be provided in the next section.

We also note that many of the specific relationships presented above are somewhat heuristic in nature, and other studies, for example those of Russo et al. (2014) or Miller and Bellan (1999), use slightly different formulations for expressions such as equations 10 or 18. As mentioned above, we use these expressions because are ultimately concerned with saline droplets specifically, but model sensitivity studies have shown that many of these specific relationships can be interchanged without significant $(<10 \%)$ quantitative effects. This is in general agreement with other model comparison studies (Miller et al., 1998). Moreover, the qualitative processes and mechanisms governing two-way thermodynamic exchange between the carrier and dispersed phases, which are the primary focus of this research, remain independent of many of these specific formulations.

\subsection{Numerical experiments}

Turbulent planar Couette flow develops between two parallel plates moving at equal and opposite velocities, as illustrated in figure 1, and as described above, Dirichlet conditions are specified for temperature and humidity at both plates. The working humidity variable is the specific humidity $q$, but boundary 


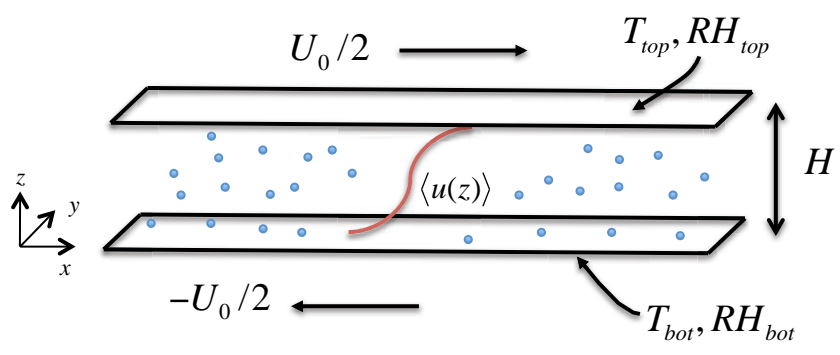

Figure 1: Schematic of Couette cell geometry. The velocity $U_{0}$ represents the velocity difference between the plates, and the Dirichlet conditions on temperature and relative humidity are denoted as $T_{t o p}, R H_{t o p}, T_{b o t}$, and $R H_{b o t}$. The distance between the plates is $H$. An example mean velocity profile is shown in red.

conditions are specified in terms of the relative humidity $R H$ determined at the temperature of the top and bottom domain boundaries using the definition of relative humidity for an ideal gas with $\rho_{a}=$ constant:

$$
R H \equiv \frac{e}{e_{s a t}}=\frac{\rho_{v}}{\rho_{v, s a t}}=\frac{q}{q_{s a t}}
$$

where $e$ is the vapor pressure, $e_{\text {sat }}$ is the saturated vapor pressure determined at a specified temperature using equation 11 , and $q_{s a t}$ and $\rho_{v, \text { sat }}$ are the corresponding saturated specific humidity and vapor density.

The primary focus of the present study is to analyze the flux of energy across the domain in the presence of evaporating droplets, including how energy transfer is partitioned under the influence of thermodynamically coupled particles. To do this, eight different simulations are performed which are used to understand droplet-induced flux modifications under (1) varying boundary forcing and (2) varying droplet size. These simulations are outlined in table 2.

With an initial mass fraction of $\phi_{i}=0.1$ and an initial droplet diameter of $d_{p, i}=80 \mu \mathrm{m}$, case 1 is designed to replicate a situation where droplets are suspended in a layer with warm, relatively moist conditions occurring below cooler, drier conditions above. Case 2 then removes the temperature difference between the top and bottom plate and reveals the effects of evaporative cooling on energy transfer. Case 3 reverses the relative humidity of case 1 in order to 


\begin{tabular}{c|cccccccc} 
Case & $\phi_{i}$ & $d_{p, i}$ & $S t_{K, i}$ & $T_{\text {top }}$ & $T_{b o t}$ & $R H_{\text {top }}$ & $R H_{b o t}$ & $\Delta h$ \\
& - & $\mu \mathrm{m}$ & - & {[]$^{\circ} \mathrm{C}$} & {[]$^{\circ} \mathrm{C}$} & $\%$ & $\%$ & $\mathrm{~J} \mathrm{~m}^{-3}$ \\
\hline \hline $1 \mathrm{U}$ & 0 & - & - & 25 & 27 & 95 & 100 & $1.38 \times 10^{4}$ \\
$1 \mathrm{~L}$ & 0.1 & 80 & 1.9 & 25 & 27 & 95 & 100 & $1.38 \times 10^{4}$ \\
& & & & & & & & \\
$2 \mathrm{U}$ & 0 & - & - & 27 & 27 & 95 & 100 & $3.81 \times 10^{3}$ \\
$2 \mathrm{~L}$ & 0.1 & 80 & 1.9 & 27 & 27 & 95 & 100 & $3.81 \times 10^{3}$ \\
$2 \mathrm{Ls}$ & 0.1 & 30 & 0.28 & 27 & 27 & 95 & 100 & $3.81 \times 10^{3}$ \\
$2 \mathrm{Ll}$ & 0.1 & 200 & 11.8 & 27 & 27 & 95 & 100 & $3.81 \times 10^{3}$ \\
& & & & & & & & \\
3U & 0 & - & - & 25 & 27 & 100 & 95 & $6.55 \times 10^{3}$ \\
3L & 0.1 & 80 & 1.9 & 25 & 27 & 100 & 95 & $6.55 \times 10^{3}$
\end{tabular}

Table 2: Simulation set performed for the current study. "U" refers to an unladen case, "L" refers to a droplet laden case. Suffix "s" refers to small initial droplet diameter and "l" refers to a large initial droplet diameter. Symbols as follows: $\phi_{i}$ is the initial mass fraction of droplets, $d_{p, i}$ is the initial droplet diameter, $S t_{K, i}$ is the initial droplet Stokes number based on the centerline Kolmogorov time scale, $T_{t o p}$ and $T_{b o t}$ are top and bottom boundary conditions for temperature, $R H_{t o p}$ and $R H_{b o t}$ are top and bottom boundary conditions for relative humidity, and $\Delta h$ is the total enthalpy difference between the bottom and top walls. 
force droplets to evaporate at the cooler wall and condense at the warm wall.

Case 2 is additionally performed with two different initial droplet diameters (30 $\mu \mathrm{m}$ and $200 \mu \mathrm{m})$ in order to assess the effect of droplet diameter on fluxes across the domain.

\section{Results and discussion}

In this work we are primarily concerned with the flux of sensible and latent heat across the domain, and how this is partitioned with and without the influence of evaporative droplets. For this purpose, horizontally and temporally averaged profiles of enthalpy, moisture, and temperature fluxes are compared between the cases outlined in table 2 . In all cases, temporal averaging is performed in a time range roughly between $10000<t^{+}<30000$. It should be noted that, as illustrated by Russo et al. (2014) using time series of the root-mean-squared droplet diameter, the system is only in what we term a "quasi-equilibrium" state, in that the droplet size distribution continues to evolve slowly in time during this range. This is due to the fact that the characteristic time scale for droplet size change is several orders of magnitude larger than that associated with temperature or momentum change (see for example Andreas (1995) or Mueller and Veron (2010)). Despite this, however, the rate of mass and energy exchange between phases quickly reaches a steady state (i.e., the exchange rate to/from each droplet is nearly in equilibrium even if the size distribution is not), and thus Eulerian statistics of the carrier phase do not vary appreciably in time.

Section 3.1 first provides a derivation of the components of the overall flux of enthalpy across the domain, determined by utilizing a Reynolds decomposition and averaging in the horizontal directions and time. Section 3.2 then compares these flux profiles for the three different cases outlined above, where boundary conditions are changed in a way to highlight various thermodynamic couplings. Finally section 3.3 presents results where the initial droplet size is varied, since droplet inertia plays a large role in cluster formation and turbophoresis, thus playing an equally large role in determining the efficiency of two-way coupling. 


\subsection{Flux partitioning}

If one begins with the enthalpy equation for the gas (see equation A.4 in the appendix), where $h_{\text {gas }}=\rho_{a} c_{p, a} T+\rho_{a} q\left(c_{p, v} T+h_{v}^{0}\right)$ is the moist enthalpy, a Reynolds decomposition of the temperature $T$, velocity $u_{i}$, and moisture $q$ yields after manipulation:

$$
\begin{aligned}
\frac{d}{d z} & {\left[\rho_{a} c_{p, a}\left\langle w^{\prime} T^{\prime}\right\rangle+\left(c_{p, v}\langle T\rangle+h_{v}^{0}\right) \rho_{a}\left\langle w^{\prime} q^{\prime}\right\rangle+c_{p, v} \rho_{a}\left\langle w^{\prime} T^{\prime} q^{\prime}\right\rangle\right] } \\
& -\frac{d}{d z}\left[\rho_{a} c_{p, a} \alpha \frac{d\langle T\rangle}{d z}+\left(c_{p, v}\langle T\rangle+h_{v}^{0}\right) D_{v} \rho_{a} \frac{d\langle q\rangle}{d z}+D_{v} c_{p, v} \rho_{a}\left\langle T^{\prime} \frac{d q^{\prime}}{d z}\right\rangle\right] \\
& -\left\langle S^{h, \text { conv }}\right\rangle-\left\langle S^{h, \text { evap }}\right\rangle-\left(c_{p, v}\langle T\rangle+h_{v}^{0}\right) \rho_{a}\left\langle S^{q}\right\rangle-c_{p, v} \rho_{a}\left\langle T^{\prime} S^{q}\right\rangle=0,
\end{aligned}
$$

where $\langle\cdot\rangle$ indicates both horizontal and temporal averaging, primes refer to fluctuating quantities, and gradients of averages in $x, y$, or $t$ are zero. This can be integrated and rearranged to yield an expression for the total upwards enthalpy flux $H_{\text {Total }}$ :

$$
H_{\text {Total }}=H_{q, \text { total }}(z)+H_{a, \text { total }}(z)+\chi(z)=\text { const } .
$$

where $H_{q, \text { total }}$ is the total enthalpy carried by the vapor phase and $H_{a, t o t a l}$ is the total enthalpy carried by the air phase, which are defined by:

$$
\begin{aligned}
H_{a, \text { total }} & =\rho_{a} c_{p, a}\left\langle w^{\prime} T^{\prime}\right\rangle-\rho_{a} c_{p, a} \alpha \frac{d\langle T\rangle}{d z}-\int_{0}^{z}\left\langle S^{h, \text { conv }}\right\rangle d z-\int_{0}^{z}\left\langle S^{h, \text { evap }}\right\rangle d z \\
& \equiv H_{a, \text { turb }}+H_{a, \text { diff }}+H_{a, \text { conv }}+H_{a, \text { evap }},
\end{aligned}
$$

and

$$
\begin{aligned}
H_{q, \text { total }} & =\left(c_{p, v}\langle T\rangle+h_{v}^{0}\right)\left[\rho_{a}\left\langle w^{\prime} q^{\prime}\right\rangle-D_{v} \rho_{a} \frac{d\langle q\rangle}{d z}-\int_{0}^{z} \rho_{a}\left\langle S^{q}\right\rangle d z\right] \\
& \equiv H_{q, \text { turb }}+H_{q, \text { diff }}+H_{q, \text { part }} .
\end{aligned}
$$

The cross-coupling term $\chi$ in equation 22 represents the transfer due to correlations between temperature and moisture fluctuations, and is given by:

$$
\chi=c_{p, v} \rho_{a}\left\langle w^{\prime} T^{\prime} q^{\prime}\right\rangle-D_{v} c_{p, v} \rho_{a}\left\langle T^{\prime} \frac{d q^{\prime}}{d z}\right\rangle-\int_{0}^{z} c_{p, v} \rho_{a}\left\langle T^{\prime} S^{q}\right\rangle d z
$$


(a)

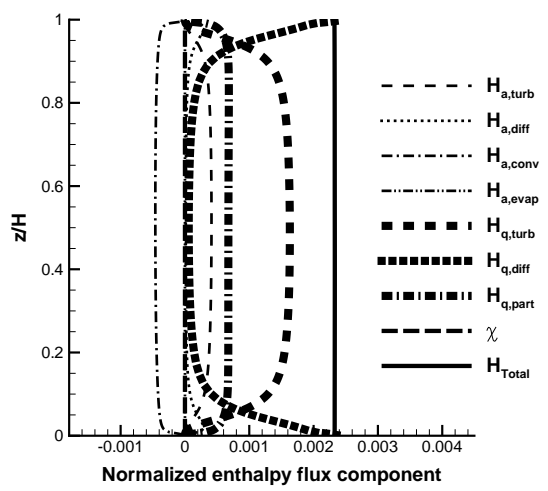

(b)

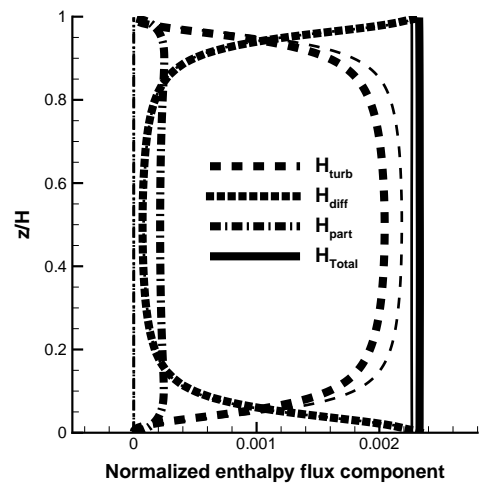

Figure 2: Flux profiles for case 1: $\left[T_{b o t}, R H_{b o t}\right]=\left[27^{\circ} C, 100 \%\right]$ and $\left[T_{t o p}, R H_{t o p}\right]=$ $\left[25^{\circ} \mathrm{C}, 95 \%\right]$. (a) Components of the vertical enthalpy flux for case 1L. Thin lines: components of $H_{a, \text { total }}$; thick lines: components of $H_{q, \text { total }}$. Line types denoted in the legend. (b) Turbulent, diffusive, and particle-induced components of $H_{\text {Total }}$ of case $1 \mathrm{~L}$ (thick lines) compared to unladen case $1 \mathrm{U}$ (thin lines). All quantities normalized by corresponding $U_{0} \Delta h$.

Thus equation 22 indicates that the total flux of enthalpy in the positive $z$ direction is uniform with height, and is a combination of the energy transported in the air phase (referred to as sensible energy), energy transported by the vapor phase (referred to as latent energy), and the cross terms. As will be shown later, $\chi$ is found to be very small, so that the total enthalpy transfer across the domain is primarily an independent sum of latent and sensible heat fluxes.

Equations 23 and 24 show that the sensible and latent energy fluxes $H_{a, t o t a l}$ and $H_{q, \text { total }}$ can be further broken down into their turbulent (subscript "turb"), diffusive (subscript "diff"), and particle-induced (subscript "part") components. It is the relative balance of each of these which will provide the bulk of the remaining discussion.

\subsection{Enthalpy fluxes}

Figure 2(a) shows the total flux $H_{\text {Total }}$ (vertical solid line), along with $\chi$ and the individual components which make up $H_{a, \text { total }}$ and $H_{q, \text { total }}$. For case 1 , where the bottom wall is warm and moist while the top wall is relatively cool 
and dry, the total enthalpy difference $\Delta h$ is larger than that for cases 2 and 3 , and most of this total difference is due to the latent heat associated with the difference in $q$ between the top and bottom plates. As a result, the total enthalpy flux is dominated by latent heat transfer - for example the total flux at the walls are nearly equal to the diffusive latent heat flux $H_{q, \text { diff }}$ (thick dotted line) at both walls, while the diffusive sensible heat flux $H_{a, \text { diff }}$ (thin dotted line) is very small. In the center of the channel, again the turbulent latent heat flux $H_{q, \text { turb }}$ is much larger than its sensible counterpart $H_{a, t u r b}$, though $H_{a, t u r b}$ is not negligible and physically represents turbulent motions bringing parcels of warm/cool air up/down across the mean temperature gradient which exists across the domain. As noted above, the cross-correlation terms contained in $\chi$ are indeed negligible compared to other components of the total flux.

The particle-induced fluxes $H_{a, \text { conv }}, H_{a, \text { evap }}$, and $H_{q, \text { part }}$ represent the enthalpy transported across the domain due to the droplets while they exchange vapor mass and heat with the surrounding flow. The evaporative sensible heat exchange $H_{a, \text { evap }}$ is negligible compared to other components, which is in agreement with the results of Russo et al. (2014). The convective sensible heat source $H_{a, c o n v}$ and the latent heat source $H_{q, \text { part }}$ meanwhile appear as fluxes of opposite sign and nearly equal magnitude across the entire channel, indicative of the fact that the droplets cool during the evaporation process. As droplets move from the warm, saturated bottom wall upwards, they begin to evaporate, which induces an upwards flux of moisture (and thus latent heat). At the same time, however, the droplet temperature decreases, and it finds itself always cooler than the surrounding air, and thus a downwards sensible heat flux is created by the particles despite being counter gradient to the imposed temperature difference. This is exactly opposite to the case of non-evaporating particles thermodynamically coupled to the flow, where the particles can enhance sensible heat transfer in the same direction as the opposed temperature gradient (Richter and Sullivan, 2014b; Kuerten et al., 2011) by carrying heat alongside the carrier phase.

The flux components in equations 23 and 24 can also be grouped according to their transport type: $H_{\text {diff }}=H_{a, \text { diff }}+H_{q, \text { diff }}, H_{\text {turb }}=H_{a, t u r b}+H_{q, \text { turb }}$, and 
(a)

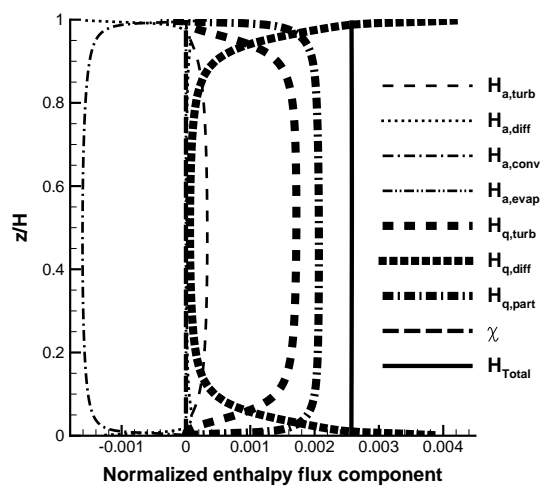

(b)

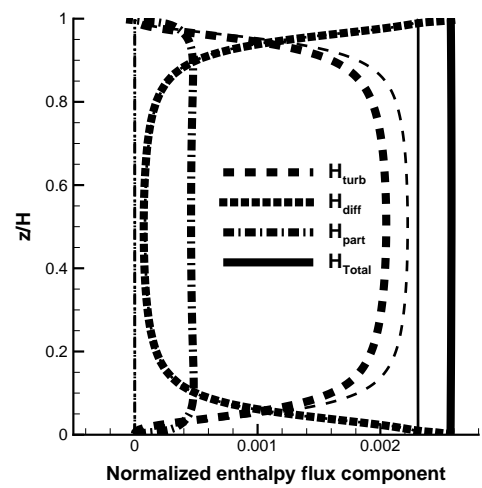

Figure 3: Same as figure 2 but for case 2: $\left[T_{b o t}, R H_{b o t}\right]=\left[27^{\circ} C, 100 \%\right]$ and $\left[T_{t o p}, R H_{t o p}\right]=$ $\left[27^{\circ} \mathrm{C}, 95 \%\right]$.

$H_{\text {part }}=H_{a, \text { conv }}+H_{a, \text { evap }}+H_{q, \text { part }}$. Figure 2(b) shows these quantities for case $1 \mathrm{~L}$ compared to the unladen case $1 \mathrm{U}$ with the same boundary conditions. Since the particle-induced fluxes are nearly equal in magnitude, figure 2(b) shows that the total particle enthalpy flux source, $H_{\text {part }}$, is relatively small compared to the total enthalpy flux across the channel. The total energy gained by an enhanced upwards transport of latent heat is nearly completely negated by an induced downwards flux of sensible heat due to evaporative cooling. Thus as compared to the unladen case, the net effect of particles is only to increase the total enthalpy flux across the channel by $3 \%$.

Figure 3 shows the same curves but for case 2, where the wall temperatures are equal while maintaining the same relative humidity difference. Thus in this case, the total enthalpy difference $\Delta h$ is entirely due to the latent heat difference since there is no net temperature difference at the walls. Figure 3(a) shows that, in contrast to figure 2, the evaporative cooling of the droplets induces a significant diffusive sensible heat flux at the walls, implying a sharp mean temperature gradient in those locations $\left(H_{a, d i f f}\right.$, thin dotted line). Similarly, a small but non-negligible turbulent sensible heat flux $H_{a, t u r b}$ is created purely through the temperature fluctuations which form as a result of particle evaporation. 
For this case, the particle-induced latent heat flux $H_{q, p a r t}$ actually exceeds the turbulent flux of latent heat $H_{q, t u r b}$, but again the particle latent heat flux is nearly cancelled by a negative particle convective heat flux $H_{a, c o n v}$, and in this case they each represent a larger fraction of $\Delta h$ than when the walls were at different temperatures in case 1 . This is due to the fact that the latent heat difference (i.e., the difference in $q$ ) is smaller in magnitude than in case 1 since the wall temperatures are equal. The evaporative sensible heat transfer is again negligible compared to other forms of energy transport.

Figure 3(b) therefore shows a similar picture as figure 2(b), where the largein-magnitude particle fluxes of latent and sensible heat mostly cancel, leaving a relatively small total particle contribution to the enthalpy flux and a corresponding increase in the total flux across the channel. The primary difference in case 2 is that, due to the smaller difference in $q$ between the plates, the particle fluxes $H_{a, c o n v}$ and $H_{q, p a r t}$ independently play a larger role, while their normalized sum ultimately plays the same role. Under these boundary conditions, the total enthalpy flux increases by $12 \%$.

Finally, figure 4 illustrates the behavior for a case where the particle-induced latent heat flux is expected to be opposite to the difference in both the carrier phase sensible and latent heat fluxes. Despite having a relative humidity of $95 \%$ at the bottom plate and $100 \%$ at the top plate, the small temperature difference results in $q_{b o t}$ exceeding $q_{t o p}:\left[q_{b o t}, q_{t o p}\right]=[0.0222,0.0209]$. As a result, the unladen case (see figure 4(b)) tends to have a net upwards flux of both sensible and latent heat. Evaporating droplets, however, highlight the nonlinearities associated with computing saturated vapor pressure, and induce a negative particle latent heat flux $H_{q, p a r t}$ (thick dash-dotted line in figure $4(\mathrm{a}))$, counter to the background $q$ gradient. Once again, however, this particleinduced latent heat flux is nearly cancelled by a now positive particle-induced sensible heat flux (since the particles now warm the flow by condensing as they travel upwards), and again they sum to a value which is a relatively small contribution to the total. In this case, this total particle contribution is now negative, and combined with the small reduction in the total turbulent flux, the 
(a)

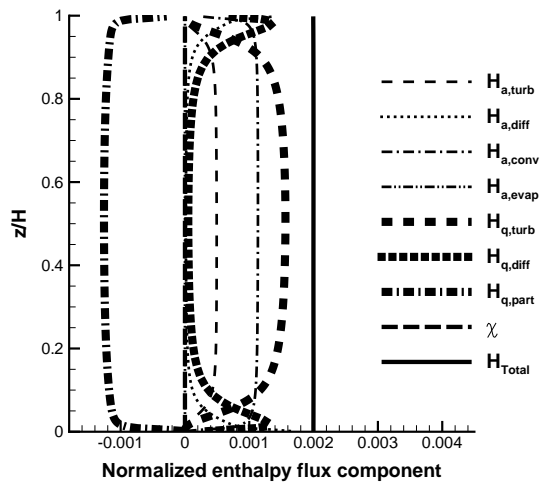

(b)

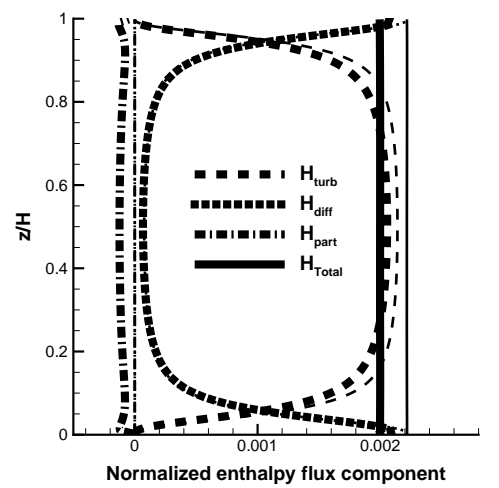

Figure 4: Same as figures 2 and 3 but for case $3: \quad\left[T_{b o t}, R H_{b o t}\right]=\left[27^{\circ} C, 95 \%\right]$ and $\left[T_{\text {top }}, R H_{\text {top }}\right]=\left[25^{\circ} \mathrm{C}, 100 \%\right]$.

total enthalpy flux across the domain reduces by $10 \%$.

Figures $2-4$ demonstrate that while evaporating droplets may have a significant influence on either moisture or sensible heat transport separately, their combined effect on total energy transfer across a domain with varying boundary forcing is small to moderate given the specified initial mass loading of $\phi_{i}=0.1$. This is generally consistent with other studies (Russo et al., 2014; Bukhvostova et al., 2014b) despite differences in boundary conditions and numerical setups. Also in agreement with previous studies of non-evaporating particles (Zonta et al., 2008; Kuerten et al., 2011; Richter and Sullivan, 2014b), all three cases display a reduction of the turbulent flux of energy across the system, which is due to a damping of velocity and temperature fluctuations across the domain.

The particle fluxes which can develop counter to background temperature and moisture gradients highlight the differences between the influences of evaporating and non-evaporating particles. Particle evaporation, which is driven entirely by the local relative humidity (and not the value of $q$ or $\rho_{v}$ ), can enhance (cases 1 and 2) or counter (case 3) the background moisture and latent heat transport, but this comes at the expense of sensible heat transport. In case 2 for instance, upward-traveling droplets evaporate and cool such that they in- 
duce both a background temperature gradient as well as a droplet sensible heat flux, in order to balance the increased latent heat flux occurring across the channel. Thus the total impact of evaporating droplets is both highly nonlinear and limited by the local conditions of the flow.

\subsection{Influence of droplet size}

Cases 2Ls and 2Ll probe the influence of droplet size on modified enthalpy transport across the domain. As demonstrated by Richter and Sullivan (2014b) and Zonta et al. (2008), the clustering of particles in near-wall turbulence heavily characterizes their collective ability to modify heat transfer in non-evaporating conditions. Depending on the particle Stokes number, droplets are able to efficiently concentrate in ejection regions near the wall, which in unladen cases would be the motions primarily responsible for turbulent heat and momentum transport (Richter and Sullivan, 2014a). Here we compare the original case with $S t_{K, i}=1.9$ to particles with $S t_{K, i}=12$ and $S t_{K, i}=0.28$. Previous studies (Richter and Sullivan, 2013, 2014a) have shown that particles with $S t_{K}=O(10)$ have the tendency to accumulate in regions associated with the large-scale Couette structures present in the domain, while $S t_{K}=O(1)$ particles tend to accumulate in the smaller-scale low-speed streaks near the wall which are associated with sweep and ejection events. Lower and higher Stokes numbers result in no appreciable local preferential accumulation.

As evidence of preferential concentration, figure 5 shows the difference between the average humidity profile $\langle q\rangle$ and the mean humidity evaluated at the particle locations $\left\langle q_{a}\right\rangle$. The quantity $\left\langle q_{a}\right\rangle$ is computed by taking the numerical average of all individual values of $q_{a}$ for all particles within slabs spanning the domain horizontally and having a height $\Delta z$ at each vertical grid point. Recall from equation 8 that the difference between $q_{*}$ and $q_{a}$ determines whether or not a particle will condense or evaporate.

Figure 5 shows that near the saturated bottom wall, the value of $\left\langle q_{a}\right\rangle$ is larger than the value of $\langle q\rangle$ (except at the first grid point above the wall), indicating that in the bottom half of the domain droplets tend to situate themselves in 


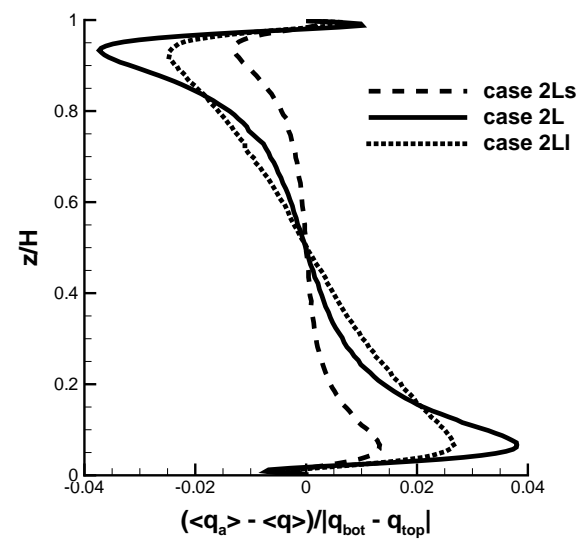

Figure 5: The difference between the average air humidity $\left\langle q_{a}\right\rangle$ evaluated at the droplet locations and the horizontally averaged humidity $\langle q\rangle$, normalized by the plate difference $\mid q_{b o t}-$ $q_{t o p} \mid$, as a function of $z / H$. Cases $2 \mathrm{Ls}, 2 \mathrm{~L}$, and $2 \mathrm{Ll}$ are compared - see legend.

relatively moist regions compared to the horizontal average. These moist regions correspond to upward plumes of moist air which are responsible for the turbulent flux of $q$ upwards. The opposite is true at the dry upper wall. The magnitude of the departure of $\left\langle q_{a}\right\rangle$ from $\langle q\rangle$ is maximized for case $2 \mathrm{~L}$, where $S t_{K, i}=1.9$, in agreement with past studies (Richter and Sullivan, 2014a,b).

At the same time, however, the difference in particle size also modifies the process of turbophoresis, whereby droplets accumulate near the walls due to gradients of turbulent kinetic energy. As shown by Zonta et al. (2008) and Russo et al. (2014), this also plays a large role in assessing the degree to which droplets can modify thermodynamic properties of wall-bounded turbulent flows. Figure 6 shows the normalized mass concentration profiles for the same three cases, where it is clear that a reduction of turbophoresis at the smallest droplet size leads to a larger and more uniform concentration of these droplets throughout the domain.

Indeed, when then observing the enthalpy flux components for cases $2 \mathrm{Ls}$, $2 \mathrm{~L}$, and $2 \mathrm{Ll}$ in figure 7 , it is seen that droplets with the smallest initial diameter can substantially enhance the total enthalpy flux across the domain, while the 


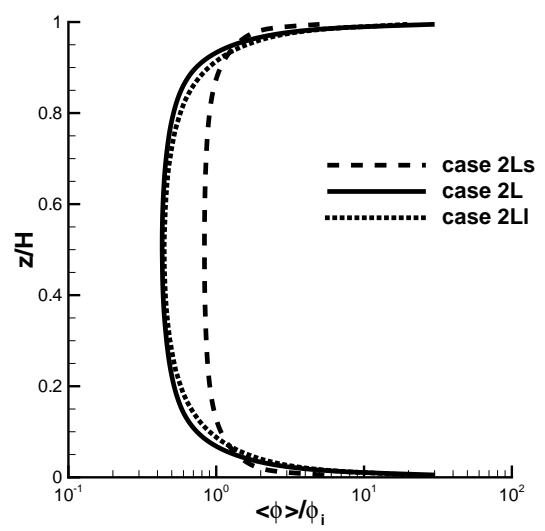

Figure 6: The average mass concentration $\langle\phi\rangle$ normalized by the initial homogeneous mass loading $\phi_{i}$ as a function of $z / H$. See legend for association with cases $2 \mathrm{Ls}, 2 \mathrm{~L}$, and $2 \mathrm{Ll}$.

largest droplets are less effective than the $S t_{K, i}=1.9$ droplets. Since the mean concentration profiles for cases $2 \mathrm{~L}$ and $2 \mathrm{Ll}$ are nearly the same, we argue that it is effectiveness in clustering which causes the differences in the modified thermodynamic fluxes observed in the system. However for case 2Ls, more droplets are available in the interior of the flow to participate in the vertical transport of sensible and latent heat, and more surface area is available for dispersed phase heat and mass transfer, overwhelming any reduction in their ability to cluster compared to case $2 \mathrm{~L}$.

\section{Conclusions}

Thermodynamically coupled evaporating droplets have been introduced into an incompressible DNS framework used to simulate turbulent Couette flow. Of particular interest is the degree to which droplets can enhance or suppress total energy transfer across the domain given Dirichlet conditions on temperature and specific humidity at the top and bottom boundaries. The microphysical model considers saline droplets, however salinity effects are negligible for the conditions studied presently. 


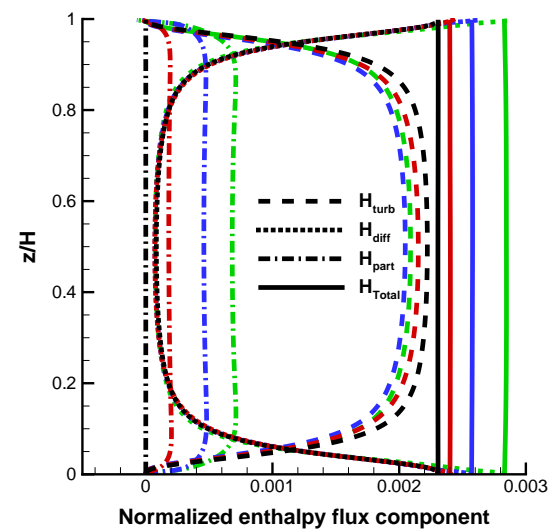

Figure 7: Comparison of turbulent $H_{\text {turb }}$ (dashed line); diffusive $H_{\text {diff }}$ (dotted line); particleinduced $H_{\text {part }}$ (dash-dotted line); and total $H_{\text {Total }}$ (solid) for all particle sizes: case $2 \mathrm{U}$ (black), case 2Ls (green), case 2L (blue), case 2Ll (red).

By varying boundary forcing, it is demonstrated that evaporating droplets have opposite effects to bulk sensible and latent heat transfer. Due to evaporative cooling, enhanced moisture transport necessarily induces an opposite-signed sensible heat flux, and the combined particle influence is thus smaller than either the sensible or latent heat flux modification considered independently. It is shown that cross-correlation terms in the flux budget are negligible and that total enthalpy transfer can be considered as a linear sum of the latent and sensible heat flux.

The effects of droplet size are probed by considering droplets of initial Stokes number $S t_{K, i}=0.28$ and $S t_{K, i}=11.8$ in addition to the base cases of $S t_{K, i}=1.9$. Between the two larger Stokes numbers, droplet clustering enhances the particle-induced energy fluxes at $S t_{K, i}=1.9$, consistent with the commonly observed peak in preferential concentration at Stokes numbers near unity (Rouson and Eaton, 2001). Smaller droplets, despite not preferentially accumulating to the same degree, substantially increase the total energy flux due to a reduction in turbophoresis and an increase in overall vapor mass transfer. 


\section{Acknowledgements}

This work was supported by the National Science Foundation under Grant No. AGS-1429921. The authors would like to thank the Computing Research Center at the University of Notre Dame for computational support and access to local resources.

\section{Appendix A. Moisture and energy coupling in the incompressible framework}

Much of the discussion contained in this appendix follows closely with the derivations presented in Russo et al. (2014), and differences between the formulations are highlighted. Throughout, all gases are assumed ideal with constant specific heats. The internal energy per unit volume $\hat{u}_{\text {gas }}$ of a parcel of moist air at temperature $T$ (in Kelvin) can be defined by:

$$
\hat{u}_{g a s}=\left(\rho_{a} c_{v, a}+\rho_{v} c_{v, v}\right) T+\rho_{v} h_{v}^{0},
$$

where $c_{v, v}$ is the specific heat of vapor at constant volume and $c_{v, a}$ is the specific heat of air at constant volume. As noted in the text, $\rho_{a}$ is the constant air density, which is assumed to be equal to the moist air ("gas") density: $\rho_{\text {gas }} \approx \rho_{a}$. The vapor density, $\rho_{v}$, is related to our working mixture variable $q=\rho_{v} / \rho_{a}$. $T$ is the air/moisture mixture temperature, and $h_{v}^{0}$ is the reference enthalpy of vapor, evaluated at $0 \mathrm{~K}$. Note that the reference state for air is in the gaseous phase at $0 \mathrm{~K}$, while that for water is in the liquid phase at $0 \mathrm{~K}$ due to the presence of liquid water droplets in the system - hence the presence of $h_{v}^{0}$.

Neglecting internal energy generation through viscous dissipation, the internal energy equation for the carrier phase can be written as:

$$
\begin{gathered}
\frac{\partial}{\partial t}\left[\left(\rho_{a} c_{v, a}+\rho_{v} c_{v, v}\right) T+\rho_{v} h_{v}^{0}\right]+\frac{\partial}{\partial x_{j}}\left[u_{j}\left(\left(\rho_{a} c_{v, a}+\rho_{v} c_{v, v}\right) T+\rho_{v} h_{v}^{0}\right)\right] \\
=k_{T} \frac{\partial^{2} T}{\partial x_{j} \partial x_{j}}-p \frac{\partial u_{j}}{\partial x_{j}}+\frac{\partial}{\partial x_{j}}\left[\left(c_{p, v} T+h_{v}^{0}\right) D_{v} \frac{\partial \rho_{v}}{\partial x_{j}}\right]+U
\end{gathered}
$$

where $k_{T}$ is the constant heat conductivity of the air, $D_{v}$ is the constant diffusivity of water vapor in air, and $p$ is the thermodynamic pressure of the air-vapor 
mixture. $U$ is the internal energy source due to the droplets which will be specified momentarily.

Upon manipulation, equation A.2 can be written as:

$$
\begin{aligned}
\rho_{a} c_{v, a} \frac{D T}{D t}+\rho_{v} c_{v, v} \frac{D T}{D t}+\left(c_{v, v} T+h_{v}^{0}\right) \frac{D \rho_{v}}{D t} \\
=k_{T} \frac{\partial^{2} T}{\partial x_{j} \partial x_{j}}+D_{v} c_{p, v} \frac{\partial T}{\partial x_{j}} \frac{\partial \rho_{v}}{\partial x_{j}}+\left(c_{p, v} T+h_{v}^{0}\right) D_{v} \frac{\partial^{2} \rho_{v}}{\partial x_{j} \partial x_{j}}-p \frac{\partial u_{j}}{\partial x_{j}}+U
\end{aligned}
$$

where the material derivative is given by $D / D t=\partial / \partial t+u_{j} \partial / \partial x_{j}$.

Since the vapor density is at all times a small fraction of the total density $(q=O(0.01))$, we can neglect the second term on the left hand side of equation A.3. Furthermore, in accordance with the findings of Russo et al. (2014), we also neglect the alignment term between temperature and density gradients (second term on right hand side). Finally, the pressure is expressed using Dalton's law of partial pressures as the sum of air and vapor pressure, which then allows for the standard Boussinesq procedure of relating the dilatation rate $\partial u_{j} / \partial x_{j}$ to the material derivative of temperature (see for example Kundu et al. (2012) p. 136), thereby introducing the specific heats at constant pressure on the left hand side:

$$
\rho_{a} c_{p, a} \frac{D T}{D t}+\left(c_{p, v} T+h_{v}^{0}\right) \frac{D \rho_{v}}{D t}=k_{T} \frac{\partial^{2} T}{\partial x_{j} \partial x_{j}}+\left(c_{p, v} T+h_{v}^{0}\right) D_{v} \frac{\partial^{2} \rho_{v}}{\partial x_{j} \partial x_{j}}+U .
$$

Equation A.4 now represents the change in gas enthalpy given the aforementioned approximations, where the total gas enthalpy $h_{\text {gas }}$ is defined as:

$$
h_{g a s}=\rho_{a} c_{p, a} T+\rho_{v}\left(c_{p, v} T+h_{v}^{0}\right) .
$$

To arrive at an equation governing the air temperature $T$, the vapor mass conservation equation (equation 3 ) is substituted for $D \rho_{v} / D t$, yielding:

$$
\rho_{a} c_{p, a} \frac{D T}{D t}=k_{T} \frac{\partial^{2} T}{\partial x_{j} \partial x_{j}}+U-\left(c_{p, v} T+h_{v}^{0}\right) S^{q},
$$

Finally, we specify $U$, which is a combination of the convective sensible heat 
transfer and vapor enthalpy exchanged at a droplet surface:

$$
U=-\sum_{\beta=1}^{\mathcal{N}_{p}} \frac{w_{\beta}}{\Delta V}\left(\dot{Q}_{c o n v}+\dot{m} h_{v, s}\right)_{\beta},
$$

where as stated in the text, $\mathcal{N}_{p}$ refers to the particles lying in the vicinity of a computational node and $w_{\beta}$ is a particle's linear weight associated with that node. The newly exchanged vapor contains enthalpy $h_{v, s}$ and is computed by assuming that the evaporation/condensation is occurring at the droplet temperature:

$$
h_{v, s}=c_{p, v} T_{p}+h_{v}^{0}
$$

Thus, when combining the last two terms of equation A.6, we arrive at equation 4:

$$
\frac{\partial T}{\partial t}+u_{j} \frac{\partial u_{j}}{\partial x_{j}}=\alpha \frac{\partial^{2} T}{\partial x_{j} \partial x_{j}}-\frac{1}{\rho_{a} c_{p, a}} \sum_{\beta=1}^{\mathcal{N}_{p}} \frac{w_{\beta}}{\Delta V}\left(\dot{Q}_{c o n v}+\dot{m} c_{p, v} T_{a}-\dot{m} c_{p, v} T_{p}\right)_{\beta} .
$$




\section{References}

Alduchov, O. A., Eskridge, R. E., 1996. Improved Magnus form approximation of saturation vapor pressure. Journal of Applied Meteorology 35 (4), 601-609.

Andreas, E. L., 1995. The temperature of evaporating sea spray droplets. Journal of the Atmospheric Sciences 52, 852-862.

Balachandar, S., Eaton, J. K., 2010. Turbulent dispersed multiphase flow. Annual Review of Fluid Mechanics 42, 111-133.

Bernardini, M., Pirozzoli, S., Orlandi, P., 2013. The effect of large-scale turbulent structures on particle dispersion in wall-bounded flows. International Journal of Multiphase Flow 51, 55-64.

Boivin, M., Simonin, O., Squires, K. D., 1998. Direct numerical simulation of turbulence modulation by particles in isotropic turbulence. Journal of Fluid Mechanics 375, 235-263.

Bukhvostova, A., Russo, E., Kuerten, J. G. M., Geurts, B. J., 2014a. Comparison of DNS of compressible and incompressible turbulent droplet-laden heated channel flow with phase transition. International Journal of Multiphase Flow $63,68-81$.

Bukhvostova, A., Russo, E., Kuerten, J. G. M., Geurts, B. J., 2014b. DNS of turbulent droplet-laden heated channel flow with phase transition at different initial relative humidities. International Journal of Heat and Fluid Flow 50, $445-455$.

Clift, R., Grace, J. R., Weber, M. E., 1978. Bubbles, Drops, and Particles. Academic Press.

Crowe, C. T., 2000. On models for turbulence modulation in fluid-particle flows. International Journal of Multiphase Flow 26 (5), 719-727. 
Dritselis, C. D., Vlachos, N. S., 2011. Numerical investigation of momentum exchange between particles and coherent structures in low Re turbulent channel flow. Physics of Fluids 23, 025103.

Elghobashi, S., 1994. On predicting particle-laden turbulent flows. Applied Scientific Research 52 (4), 309-329.

Elghobashi, S., Truesdell, G. C., 1993. On the two-way interaction between homogeneous turbulence and dispersed solid particles. I: Turbulence modification. Physics of Fluids A: Fluid Dynamics 5, 1790.

Ferrante, A., Elghobashi, S., 2003. On the physical mechanisms of two-way coupling in particle-laden isotropic turbulence. Physics of Fluids 15 (2), 315329 .

Kaftori, D., Hetsroni, G., Banerjee, S., 1998. The effect of particles on wall turbulence. International Journal of Multiphase Flow 24 (3), 359-386.

Komminaho, J., Lundbladh, A., Johansson, A. V., 1996. Very large structures in plane turbulent Couette flow. Journal of Fluid Mechanics 320, 259-285.

Kuerten, J. G. M., van der Geld, C. W. M., Geurts, B. J., 2011. Turbulence modification and heat transfer enhancement by inertial particles in turbulent channel flow. Physics of Fluids 23, 123301.

Kuerten, J. G. M., Vreman, A. W., 2015. Effect of droplet interaction on dropletladen turbulent channel flow. Physics of Fluids 27, 053304.

Kulick, J. D., Fessler, J. R., Eaton, J. K., 1994. Particle response and turbulence modification in fully developed channel flow. Journal of Fluid Mechanics 277, $109-134$.

Kundu, P. K., Cohen, I. M., Dowling, D. R., 2012. Fluid Mechanics, 5th Edition. Academic Press, Boston.

Lee, J., Lee, C., 2015. Modification of particle-laden near-wall turbulence: Effect of Stokes number. Physics of Fluids 27, 023303. 
Marchioli, C., Soldati, A., Kuerten, J. G. M., Arcen, B., Taniere, A., Goldensoph, G., Squires, K. D., Cargnelutti, M. F., Portela, L. M., 2008. Statistics of particle dispersion in direct numerical simulations of wall-bounded turbulence: Results of an international collaborative benchmark test. International Journal of Multiphase Flow 34, 879-893.

Mashayek, F., 1998. Droplet-turbulence interactions in low-Mach-number homogeneous shear two-phase flows. Journal of Fluid Mechanics 367, 163-203.

Maxey, M. R., Riley, J. J., 1983. Equation of motion for a small rigid sphere in nonuniform flow. Physics of Fluids 26 (4), 883-889.

Miller, R. S., Bellan, J., 1999. Direct numerical simulation of a confined threedimensional gas mixing layer with one evaporating hydrocarbon-droplet-laden stream. Journal of Fluid Mechanics 384, 293-338.

Miller, R. S., Harstad, K., Bellan, J., 1998. Evaluation of equilibrium and nonequilibrium evaporation models for many-droplet gas-liquid flow simulations. International Journal of Multiphase Flow 24, 1025-1055.

Mito, Y., Hanratty, T. J., 2006. Effect of feedback and inter-particle collisions in an idealized gas-liquid annular flow. International Journal of Multiphase Flow 32, 692-716.

Mueller, J. A., Veron, F., 2010. A Lagrangian stochastic model for sea-spray evaporation in the atmospheric marine boundary layer. Boundary-Layer Meteorology 137, 135-152.

Pruppacher, H. R., Klett, J. D., 1997. Microphysics of clouds and precipitation, 2nd Edition. Kluwer Academic Publishers.

Ranz, W. E., Marshall, W. R., 1952. Evaporation from drops. Chemical Engineering Progress 48, 141-146.

Richter, D. H., 2015. Turbulence modification by inertial particles and its influence on the spectral energy budget in planar Couette flow. Physics of Fluids $27,063304$. 
Richter, D. H., Sullivan, P. P., 2013. Momentum transfer in a turbulent, particleladen Couette flow. Physics of Fluids 25, 053304.

Richter, D. H., Sullivan, P. P., 2014a. Modification of near-wall coherent structures by inertial particles. Physics of Fluids 26, 103304.

Richter, D. H., Sullivan, P. P., 2014b. The sea spray contribution to sensible heat flux. Journal of the Atmospheric Sciences 71 (2), 640-654.

Righetti, M., Romano, G. P., 2004. Particle-fluid interactions in a plane nearwall turbulent flow. Journal of Fluid Mechanics 505, 93-121.

Rouson, D. W. I., Eaton, J. K., 2001. On the preferential concentration of solid particles in turbulent channel flow. Journal of Fluid Mechanics 428, 149-169.

Russo, E., Kuerten, J. G. M., van der Geld, C. W. M., Geurts, B. J., 2014. Water droplet condensation and evaporation in turbulent channel flow. Journal of Fluid Mechanics 749, 666-700.

Sardina, G., Schlatter, P., Brandt, L., Picano, F., Casciola, C. M., Apr. 2012. Wall accumulation and spatial localization in particle-laden wall flows. Journal of Fluid Mechanics 699, 50-78.

Shotorban, B., Mashayek, F., Pandya, R. V. R., 2003. Temperature statistics in particle-laden turbulent homogeneous shear flow. International Journal of Multiphase Flow 29, 1333-1353.

Soldati, A., Marchioli, C., 2009. Physics and modelling of turbulent particle deposition and entrainment: Review of a systematic study. International Journal of Multiphase Flow 35, 827-839.

Spalart, P. R., Moser, R. D., Rogers, M. M., 1991. Spectral methods for the Navier-Stokes equations with one infinite and two periodic directions. Journal of Computational Physics 96, 297-324. 
Vaillancourt, P. A., Yau, M. K., 2000. Review of particle-turbulence interactions and consequences for cloud physics. Bulletin of the American Meteorological Society 81 (2), 285-298.

Yamamoto, Y., Potthoff, M., Tanaka, T., Kajishima, T., Tsuji, Y., 2001. Largeeddy simulation of turbulent gas-particle flow in a vertical channel: effect of considering inter-particle collisions. Journal of Fluid Mechanics 442, 303-334.

Zhao, L., Andersson, H. I., Gillissen, J. J. J., 2013. Interphasial energy transfer and particle dissipation in particle-laden wall turbulence. Journal of Fluid Mechanics 715, 32-59.

Zonta, F., Marchioli, C., Soldati, A., 2008. Direct numerical simulation of turbulent heat transfer modulation in micro-dispersed channel flow. Acta Mechanica 195, 305-326.

Zonta, F., Marchioli, C., Soldati, A., 2011. Time behavior of heat fluxes in thermally coupled turbulent dispersed particle flows. Acta Mechanica 218, $367-373$.

Zonta, F., Marchioli, C., Soldati, A., 2013. Particle and droplet deposition in turbulent swirled pipe flow. International Journal of Multiphase Flow 56, $172-183$.

Zonta, F., Onorato, M., Soldati, A., 2012. Turbulence and internal waves in stably-stratified channel flow with temperature-dependent fluid properties. Journal of Fluid Mechanics 697, 175-203.

Zonta, F., Soldati, A., 2014. Effect of temperature dependent fluid properties on heat transfer in turbulent mixed convection. Journal of Heat Transfer 136, 022501. 